\title{
Depth dependence of the ionization energy of shallow hydrogen states in $\mathrm{ZnO}$ and $\mathrm{CdS}$
}

\author{
T. Prokscha, ${ }^{1, *}$ H. Luetkens, ${ }^{1}$ E. Morenzoni, ${ }^{1}$ G. J. Nieuwenhuys,${ }^{1,2, \dagger}$ A. Suter, ${ }^{1}$ M. Döbelii, ${ }^{3}$ \\ M. Horisberger, ${ }^{4}$ and E. Pomjakushina ${ }^{4}$ \\ ${ }^{1}$ Laboratory for Muon Spin Spectroscopy, Paul Scherrer Institut, CH-5232 Villigen PSI, Switzerland \\ ${ }^{2}$ Kamerlingh Onnes Laboratory, Leiden University, 2300 RA Leiden, The Netherlands \\ ${ }^{3}$ Ion Beam Physics, ETH Zurich, $\mathrm{CH}-8093$ Zurich, Switzerland \\ ${ }^{4}$ Laboratory for Developments and Methods, Paul Scherrer Institut, CH-5232 Villigen PSI, Switzerland \\ (Received 29 August 2014; revised manuscript received 18 November 2014; published 1 December 2014)
}

\begin{abstract}
The characteristics of shallow hydrogenlike muonium $(\mathrm{Mu})$ states in nominally undoped $\mathrm{ZnO}$ and $\mathrm{CdS}(0001)$ crystals have been studied close to the surface at depths in the range of 10-180 nm by using low-energy muons and in the bulk using conventional $\mu \mathrm{SR}$. The muon implantation depths are adjusted by tuning the energy of the low-energy muons between 2.5 and $30 \mathrm{keV}$. We find that the bulk ionization energy $E_{i}$ of the shallow donorlike $\mathrm{Mu}$ state is lowered by about $10 \mathrm{meV}$ at a depth of $100 \mathrm{~nm}$, and continuously decreasing on approaching the surface. At a depth of about $10 \mathrm{~nm}, E_{i}$ is further reduced by $25-30 \mathrm{meV}$ compared to its bulk value. We attribute this change to the presence of electric fields due to band bending close to the surface, and we determine the depth profile of the electric field within a simple one-dimensional model.
\end{abstract}

DOI: 10.1103/PhysRevB.90.235303

\section{INTRODUCTION}

For future semiconductor technologies, the incorporation, profiling, and monitoring of dopants are considered to be key issues for novel device applications [1]. The binding energy of a dopant is an important characteristic, and recent studies have focused on the investigation of the binding energies of single hydrogenic defect states close to semiconductor surfaces, in nanoscale devices, or in quantum wells [2-5]. For a shallow Coulombic hydrogenic impurity state in the effective mass approximation, the binding energy of the state is predicted to decrease when approaching a potential barrier at a semiconductor interface or surface [6,7]. A model proposed by Levine [8] showed that at a semiconductor surface the ground state of a shallow impurity is the $2 p$ state, which means that the binding energy of the surface donor is $1 / 4$ of the bulk donor. However, image charges at the surface cause the binding energy to be closer to its bulk value, while still being reduced [9]. In contrast, recent theoretical studies found an increase of the hydrogenic impurity binding energies in nanowires and quantum dots due to dielectric confinement $[10,11]$. Additionally, an experimental investigation of $\mathrm{Si}$ doped GaAs estimated an increasing binding energy at depths $z<1.5 \mathrm{~nm}$ [5], and the authors concluded that the effective mass approach will fail for all hydrogenic donors close to a semiconductor surface.

The descibed effects so far occur on a length scale of typically less than ten nanometers. In this paper, we will discuss the change of the binding energy of single shallow hydrogenlike donor states on a much larger length scale at depths between $10 \mathrm{~nm}$ and $\sim 200 \mathrm{~nm}$, where the effective mass approximation appears to be appropriate, and dielectric confinement and position dependent effective masses or dielectric constants are negligible. Even in the case of band bending at the surface-which is of particular importance for

\footnotetext{
*thomas.prokscha@psi.ch

${ }^{\dagger}$ Deceased.
}

PACS number(s): 71.55.Gs, 73.40.Vz, 61.72.uj, 76.75.+i

this work-the effective mass approximation is expected to hold because the fractional change of the perturbation potential over the dimension of a unit cell is negligibly small $[12,13]$ (less than $1 \%$ in our case). In case of good sample quality with dislocation line densities $\lesssim 10^{5} \mathrm{~cm}^{-2}$, the effect of internal strain, due to these dislocations, on the ionization energy of shallow donor states can be neglected [14]. However, the donor ionization energy may be affected by the presence of electric fields close to the sample surface: in a lowly doped semiconductor, surface states can cause a pinning of the Fermi level at the surface, which results in a band bending on a length scale given by the Debye length [15]. In this case, the solution of the Poisson equation yields an quadratic $z$ dependence of the electrostatic potential in the band bending zone close to the surface [15], which means a linearly increasing electric field towards the surface. The presence of an electric field lowers the ionization energy of the shallow impurity (Poole-Frenkel effect in insulators or semiconductors [15-18]), and since the electric field increases on approaching the surface, the binding energy is expected to decrease when getting closer to the surface.

Hydrogen as an ubiquitous impurity is of particular interest in semiconductor technology, because it often modifies the electrical and optical properties in an unwanted way due to its amphoteric behavior, which may cause doping counteracting the prevailing type of conductivity. The characterization of hydrogen impurities in semiconductors is often difficult, particularly if one wants to study single (or solitary) dopants. Here, positively charged muons $\left(\mu^{+}\right)$can help to circumvent these difficulties. Muons played an important role in the identification and characterization of isolated hydrogen defect centers in semiconductors [19-22]. Implanted in a semiconductor or insulator the $\mu^{+}$stops at an interstitial site, and may capture one or two electrons to form the light hydrogen pseudoisotope muonium $\left[\mathrm{Mu},\left(\mu^{+} e^{-}\right)\right.$, mass of $\mu^{+} \simeq 1 / 9$ proton masses]. Depending on the concentration of other dopants, and on $\mathrm{Mu}$ formation energy, it occurs in either of three charge states $\mathrm{Mu}^{+}, \mathrm{Mu}^{0}$, or $\mathrm{Mu}^{-}$, analogous to hydrogen. The neutral state can be spectroscopically distinguished from the charged states in muon spin rotation experiments $(\mu \mathrm{SR})$ 
[23]. The recent theoretical discovery of a universal alignment of the so-called hydrogen pinning level $\varepsilon(+/-)$ - where the formation energies of the positive and negative impurity are equal-allows predictions whether hydrogen forms a shallow donor: this occurs if the pinning level is close to or above the conduction band minimum [24,25]. The predicted shallow hydrogen donor states in $\mathrm{ZnO}$ [26] and InN [25] have been first confirmed by $\mu \mathrm{SR}$ measurements [27,28], closely followed in $\mathrm{ZnO}$ by EPR [29] and infrared spectroscopy [30,31]. In $\mathrm{CdS}$, the observed shallow $\mathrm{Mu}$ state [32,33] does not necessarily imply, according to the theoretical models, that in thermodynamic equilibrium hydrogen acts as a shallow donor. Since the muon experiments take place on a microsecond time scale (muon life time is $\sim 2.2 \mu \mathrm{s}$ ), the observed shallow $\mathrm{Mu}$ state could be a metastable state [34-36].

In this paper, we present the depth dependence of the ionization energy of the shallow $\mathrm{Mu}$ state in $\mathrm{CdS}$ and of the shallow donor state in $\mathrm{ZnO}$, and at the interface of a 20-nm-thin Au film sputtered on $\mathrm{ZnO}$. This allows us to obtain direct information about the effect of electric fields-due to band bending — on the ionization energies of the corresponding hydrogenlike states in a range of $\sim 200 \mathrm{~nm}$ beneath the surface, or at a metal-semiconductor interface. The ionization energies continuously decrease on approaching the surface/interface, reaching a reduction of $25-30 \mathrm{meV}$ at a depth of $10 \mathrm{~nm}$. We use the observed "ionization profile" to determine by a simple one-dimensional model the electric field profile at the surface/interface. This is to best of our knowledge the first time that the "ionization profile" of a single impurity and the derived electric field profile has been visualized by means of a local probe implantation technique. It offers several advantages compared to conventional experimental techniques. Photoemission spectroscopy and other surface sensitive techniques (see the review of Koenraad and Flatté [1] and references therein) are limited to a few surface layers, and cannot access interfaces at a depth of tens of nanometers. Deep-level transient spectroscopy is not applicable to lowdoped materials, shallow impurity states, and single dopants, and usually requires a $p-n$ junction. In contrast, there are no such limitations for muon spin rotation, which is contactless, and provides intrinsic information, about shallow as well as deep hydrogen states. They are incorporated as solitary dopants, and their properties can be monitored as a function of distance to the surface or an interface, thus providing a new experimental tool to address the issues raised at the beginning of this introduction.

\section{EXPERIMENTAL DETAILS}

The $\mu \mathrm{SR}$ experiments were carried out at the Swiss Muon Source $(\mathrm{S} \mu \mathrm{S}$, Paul Scherrer Institut, Villigen, Switzerland). For the near-surface measurements at mean depths $\langle z\rangle<$ $200 \mathrm{~nm}$, we used the low-energy $\mu$ SR spectrometer (LE- $\mu$ SR) LEM at the $\mu \mathrm{E} 4$ beam line $[39,40]$. Polarized low-energy $\mu^{+}$ with energies in the $\mathrm{keV}$ range are generated by moderation of a 4-MeV muon beam in a solid, about 250-nm-thin Ar film with a $\sim 10$-nm-thin $\mathrm{N}_{2}$ capping layer, deposited at $10 \mathrm{~K}$ on a patterned $\mathrm{Ag}$ foil [41-44]. Epithermal muons with a mean energy of $\sim 15 \mathrm{eV}$ escape into vacuum with a conversion efficiency from MeV-to-eV of $\sim 5 \times 10^{-5}$. They
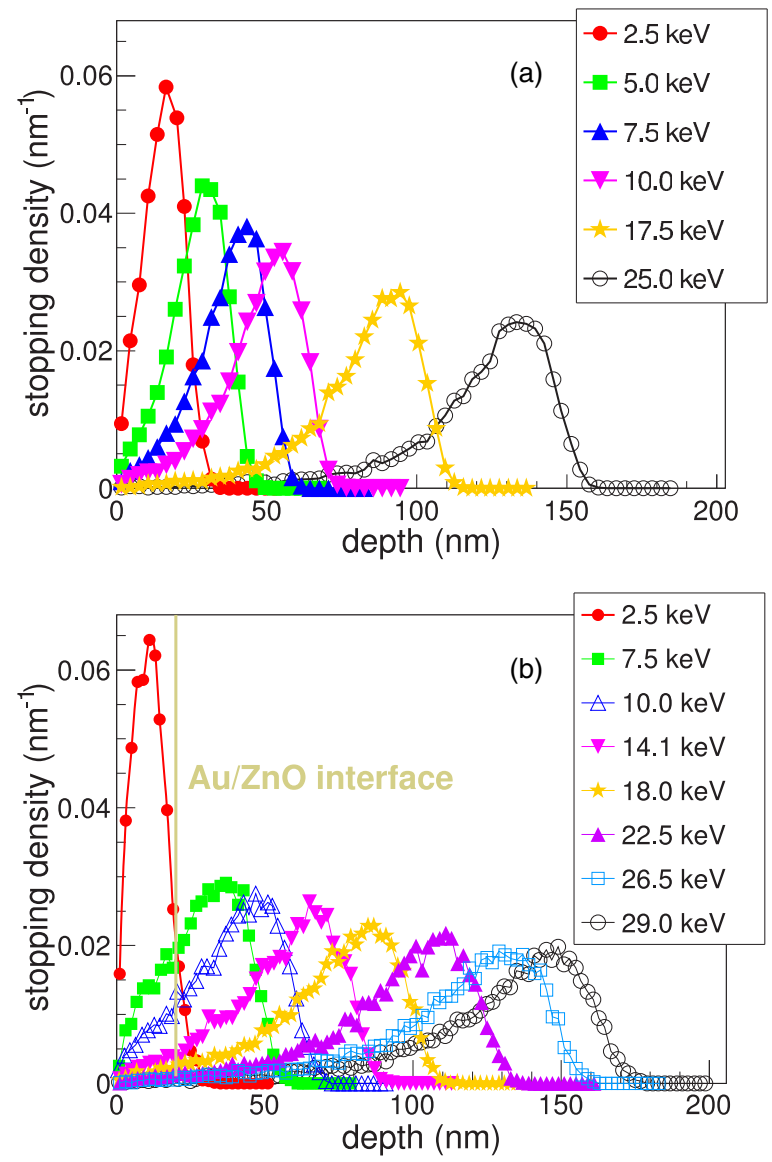

FIG. 1. (Color online) Simulated muon implantation profiles in (a) $\mathrm{ZnO}$ and (b) $20-\mathrm{nm} \mathrm{Au}$ on top of $\mathrm{ZnO}$, using the program TRIMSP [37], which has been shown to calculate the stopping profile with sufficient accuracy [38].

are accelerated electrostatically to energies up to $20 \mathrm{keV}$ by applying a positive bias to the Ag moderator foil, and then transported by use of electrostatic elements over a distance of about two meters to the sample cryostat. The muon implantation energy was varied between 2.5 and $30 \mathrm{keV}$, corresponding to mean implantation depths of 10 and $150 \mathrm{~nm}$, respectively, see Fig. 1. The implantation energy is usually varied by applying a positive or negative bias of up to $12 \mathrm{kV}$ to the electrically insulated sample holder [39]. Shallow $\mathrm{Mu}$ formation deep in the bulk at $\langle z\rangle \sim 200-300 \mu \mathrm{m}$ was studied at the GPS spectrometer at the $\pi \mathrm{M} 3$ beam line with a muon beam energy of about $3.5 \mathrm{MeV}$.

The samples were nominally undoped $\mathrm{ZnO}$ and $\mathrm{CdS}$ wafers [(0001) orientation, supplier: Crystec $\mathrm{GmbH}$, Berlin, Germany; resistivity $>10 \Omega \mathrm{cm}$ and $>1 \mathrm{k} \Omega \mathrm{cm}$, respectively]. The $\mathrm{ZnO}$ crystals had a size of $10 \times 10 \mathrm{~mm}^{2}$ and a thickness of $0.5 \mathrm{~mm}$. Nine pieces were glued with conductive silver onto a standard sample plate of LEM. This mosaic of samples ensured that no muons missed the sample, therefore eliminating any background contribution (the low-energy muon beam spot has a FWHM of about $13 \mathrm{~mm}$ ). In the second experiment, several pieces of the $\mathrm{ZnO}$ crystals were sputtered with a 20-nm-thin $\mathrm{Au}$ film to study any changes introduced by the Schottky barrier at the $\mathrm{Au} / \mathrm{ZnO}$ interface. The CdS sample was one half of a 2 " wafer, $0.5-\mathrm{mm}$ thick, which was also large enough to stop all 
muons in the sample. All samples were polished on both sides. For the GPS measurements, one of the $\mathrm{ZnO}$ crystals was used, and a $\sim 5 \times 10 \mathrm{~mm}^{2}$ broken-off piece of the CdS wafer.

Transverse field $\mu$ SR measurements have been performed with a magnetic field of $10-\mathrm{mT}$ applied parallel to the $\langle 0001\rangle$ direction, and the muon spin initially parallel to the sample surface at LEM, and out of plane at GPS. Shallow Mu in $\mathrm{CdS}$ and $\mathrm{ZnO}$ has an anistropic, axially symmetric hyperfine interaction with the hyperfine coupling constants $A_{\|}$and $A_{\perp}$ parallel and perpendicular to the symmetry axis, which is along the $\mathrm{Cd}-\mathrm{S}$ or the $\mathrm{Zn}-\mathrm{O}$ bond direction $[32,33]$. In the high-field limit, two shallow $\mathrm{Mu}$ lines can be observed with a separation $\Delta v(\Theta)=A_{\|} \cos ^{2} \Theta+A_{\perp} \sin ^{2} \Theta$, where $\Theta$ is the angle between the Mu symmetry axis and the externally applied field. The two lines are placed symmetrically around the "central" line, i.e., the precession frequency of bare $\mu^{+}$ without bound electron. In the geometry of the experiment, there is one shallow $\mathrm{Mu}$ state at a bond parallel to the $\langle 0001\rangle$ direction $(\Theta=0)$, and three $\mathrm{Mu}$ states at the bonds under $\Theta=109.4^{\circ}$ with respect to the $\langle 0001\rangle$ axis. This leads to two pairs of $\mathrm{Mu}$ lines with an intensity ratio of 1:3. For $\mathrm{ZnO}$ and $\mathrm{CdS}$, the hyperfine couplings are $A_{\|}=760(30)$ and $335(8) \mathrm{kHz}, A_{\perp}=370(22)$ and $199(6) \mathrm{kHz}$, respectively [33]. However, only in $\mathrm{CdS}$, the $\mathrm{Mu}$ lines are narrow and well resolved in bulk $\mu \mathrm{SR}$ experiments whereas in $\mathrm{ZnO}$, spin and/or charge exchange with impurities or free charge carriers even at low temperatures $(5 \mathrm{~K})$ lead to a sizable broadening of the $\mathrm{Mu}$ lines, which smears out the $\mathrm{Mu}$ satellite lines [32,33]. In $\mathrm{CdS}$, the spitting of the inner lines $\left(\Theta=109.4^{\circ}\right)$ is $\Delta \nu_{\mathrm{I}}=$ $0.214(5) \mathrm{MHz}$, and the splitting of the outer lines $\left(\Theta=0^{\circ}\right)$ is $v_{\mathrm{O}}=0.335(8) \mathrm{MHz}$. In $\mathrm{ZnO}$, the correponding separations are $\Delta v_{\mathrm{I}}=0.413(20) \mathrm{MHz}$, and $\Delta v_{\mathrm{O}}=0.760(30) \mathrm{MHz}$.

The ratio of ionized to neutral impurities (donors) as a function of temperature $T$ is given by [45]

$$
\frac{N_{D}^{+}}{N_{D}^{0}}=\frac{N_{c}}{n} \frac{1}{g_{D}} \exp \left(-E_{i} / k_{B} T\right) \equiv N \exp \left(-E_{i} / k_{B} T\right),
$$

where $N_{c}$ is the effective density of states in the conduction band, $n$ is the concentration of free carriers, $g_{D}$ is the impurity spin degeneracy, $E_{i}$ is the ionization energy of the donor, and $N$ is a density-of-states parameter. We can rewrite in terms of ionized $(f)$ and unionized $(1-f)$ muonium fractions [46]: $f /(1-f)=N_{D}^{+} / N_{D}^{0}$. With this it follows for the neutral $\mathrm{Mu}^{0}$ fraction $f_{\mathrm{Mu}}(T)$ :

$$
f_{\mathrm{Mu}}(T)=[1-f(T)]=\frac{1}{1+N \exp \left(-E_{i} / k_{B} T\right)} .
$$

Thus, by measuring the neutral $\mathrm{Mu}$ fraction $f_{\mathrm{Mu}}(T)$ as a function of temperature, the donor ionization energy $E_{i}$ can be determined. In case of well resolved satellite lines, the $\mu \mathrm{SR}$ asymmetry spectra can be fit by a sum of five Lorentzians (i.e., exponential relaxation in time domain), with the sum $A_{\mathrm{Mu}}$ of asymmetries (amplitudes) of the four $\mathrm{Mu}$ satellite lines, and the asymmetry $A_{\mathrm{D}}$ of the so-called diamagnetic signal, i.e., a $\mu^{+}$without bound electron. The neutral fraction $f_{\mathrm{Mu}}(T)$ is then given by

$$
f_{\mathrm{Mu}}(T)=\frac{A_{\mathrm{Mu}}(T)}{A_{\mathrm{Mu}}(T)+A_{\mathrm{D}}(T)} .
$$
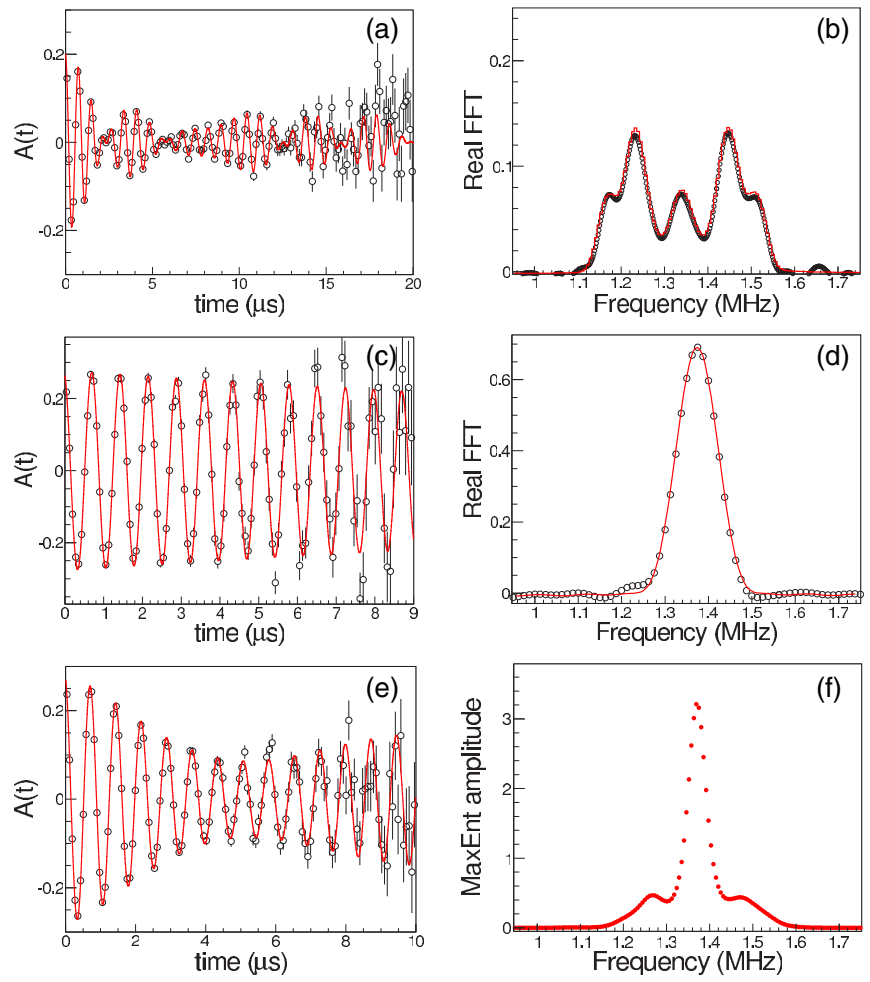

FIG. 2. (Color online) CdS (0001), 10-mT applied parallel to a $\langle 0001\rangle$ direction, $\mu \mathrm{SR}$ asymmetry spectra $A(t)$, and corresponding real part of fast Fourier transform (Real FFT). (a) and (b) $5 \mathrm{~K}$, bulk $\mu$ SR (GPS instrument, Muons-On-REquest (MORE) mode [50]) ( $\langle\mathrm{z}\rangle \sim 280 \mu \mathrm{m}$ ). (c) and (d) $5 \mathrm{~K}$, implantation energy $25 \mathrm{keV}$, virgin polished sample ( $\langle\mathrm{z}\rangle \sim 140 \mathrm{~nm}$ ). (e) and (f) $5 \mathrm{~K}$, implantation energy 23-26.5 keV, etched sample. The spectrum in (f) is obtained by a maximum entropy fit $[51,52]$ to the data in four positron counters. The solid red lines are fits to the data as described in the text, using the program MUSRFIT [53].

\section{RESULTS}

Asymmetry and corresponding frequency spectra for CdS and $\mathrm{ZnO}$ are shown in Figs. 2 and 4, respectively. The analysis has been done in the following way. In practice, it is difficult to derive the temperature dependence of the neutral fraction by trying to fit five lines to the experimental data over the whole temperature range. Problems arise in this procedure in the case of poorly resolved satellites or small $\mathrm{Mu}$ fractions. Also, with increasing temperature spin-exchange processes due to thermally activated charge carriers lead to broadening of the $\mathrm{Mu}$ satellites and a phase shift of the Mu signal with respect to the $\mu^{+}$signal $[47,48]$. This makes fits in the ionization regime more difficult: whereas at temperatures $T \lesssim 15 \mathrm{~K}$ the $\mathrm{CdS}$ and $\mathrm{ZnO}$ data could be fit with five lines, where we fixed the splitting of the $\mathrm{Mu}$ lines to the known values, this procedure didn't work well in the ionization regime. Therefore we simplified the analysis by an approximation: in $\mathrm{CdS}$ the $\mu \mathrm{SR}$ asymmetry spectra $A(t)$ were fit over the whole temperature range by the sum of two exponentially decaying components:

$$
A(t)=\left[A_{\mathrm{D}} \exp \left(-\lambda_{\mathrm{D}} t\right)+A_{\mathrm{Mu}} \exp \left(-\lambda_{\mathrm{Mu}} t\right)\right] \cos (\omega t+\phi),
$$


where $\lambda_{\mathrm{D}}$ is the relaxation rate of the central, diamagnetic line which we fixed to the high temperature value (where $\mathrm{Mu}$ is ionized, $\left.\lambda_{\mathrm{D}} \lesssim 0.01 \mu \mathrm{s}^{-1}\right), \omega=\gamma_{\mu} B$ is the $\mu^{+}$precession frequency in the applied magnetic field $B=10 \mathrm{mT}$, and $\gamma_{\mu} / 2 \pi=135.5 \mathrm{MHz} / \mathrm{T}$ is the gyromagnetic ratio of the muon, and $\phi$ is a detectors phase of the corresponding decay positron detector. In this way, the temperature dependent $\mathrm{Mu}$ fraction $f_{\mathrm{Mu}}(T)$ can be calculated according to Eq. (3), which is then used to determine the ionization energy by fitting Eq. (2) to $f_{\mathrm{Mu}}(T)$. In the case of $\mathrm{ZnO}$ and $\mathrm{Au} / \mathrm{ZnO}$ with poorly resolved satellites, we further simplified the analysis by using a single exponentially relaxing component:

$$
A(t)=A \exp (-\lambda t) \cos (\omega t+\phi) .
$$

The temperature dependence of $\lambda(T)$ can be well approximated by Eq. (2) [49]:

$$
\begin{aligned}
\lambda(T) & =f_{\mathrm{Mu}}(T) \lambda_{\mathrm{Mu}}(T)+\left[1-f_{\mathrm{Mu}}(T)\right] \lambda_{\mathrm{D}} \\
& \simeq f_{\mathrm{Mu}}(T) \lambda_{\mathrm{Mu}}(0)+\lambda_{\mathrm{D}},
\end{aligned}
$$

and we verified this procedure for $\mathrm{CdS}$ by comparing this analysis method with the two-component fits of Eq. (4): both methods yield the same ionization energies within experimental errors.

\section{A. CdS}

Muon spin rotation asymmetry and the corresponding frequency spectra at a temperature of $5 \mathrm{~K}$ of the virgin $\mathrm{CdS}$ wafer in the bulk and at $\langle z\rangle \sim 140 \mathrm{~nm}$ are shown in Figs. 2(a)-2(d). In the bulk, a clear beating is visible reflecting the presence of four shallow Mu satellite lines and the center $\mu^{+}$line of muons, which do not form shallow Mu. The two lines with smaller splitting and higher intensity are due to shallow $\mathrm{Mu}$ at the bonds under $109.4^{\circ}$ with respect to the $\langle 0001\rangle$ axis, and the two lines with larger splitting are from shallow $\mathrm{Mu}$ at the bond parallel to the $\langle 0001\rangle$ axis. In contrast to the bulk measurements the LEM data do not show any beating which means that in the near-surface region either shallow Mu does not form, or is strongly suppressed, or-due to the presence of defects-fast spin and/or charge exchange with a defect electron causes a "collapse" of the line splitting resulting in a broadening of the diamagnetic line. Recent bulk $\mu \mathrm{SR}$ experiments in $\mathrm{CdS}$ and $\mathrm{Si}$ demonstrated the reduction of the $\mathrm{Mu}$ formation probability in the presence of defects [54]. Rutherford backscattering spectrometry (RBS) channeling measurements with $2-\mathrm{MeV}$ He nuclei at the Institute of Ion Beam Physics at the ETH, Zurich, revealed that a surface layer of at least one $\mu \mathrm{m}$ depth has a high defect concentration, probably caused by the mechanical polishing of the $\mathrm{CdS}$ wafer. We attribute the absence of the characteristic shallow Mu lines and the slight broadening of the diamagnetic line in Figs. 2(c) and 2(d) to the presence of these defects. In order to remove the defect-rich surface layer the $\mathrm{CdS}$ sample was etched for $55 \mathrm{~min}$ at $60^{\circ} \mathrm{C}$ in a $1: 1 \mathrm{HCL} / \mathrm{H}_{2} \mathrm{O}$ solution. The final thickness of the wafer was $0.35(4) \mathrm{mm}$, meaning that a total of $\sim 150 \mu \mathrm{m}$ of material was removed. After this procedure, the $\mu$ SR data show the characteristic beating typical for shallow $\mathrm{Mu}$, see Figs. 2(e) and 2(f). Compared to the bulk data the shallow $\mathrm{Mu}$ fraction is clearly reduced, and the satellite lines are not resolved due to the shorter time window of $10 \mu \mathrm{s}$ in LEM. The

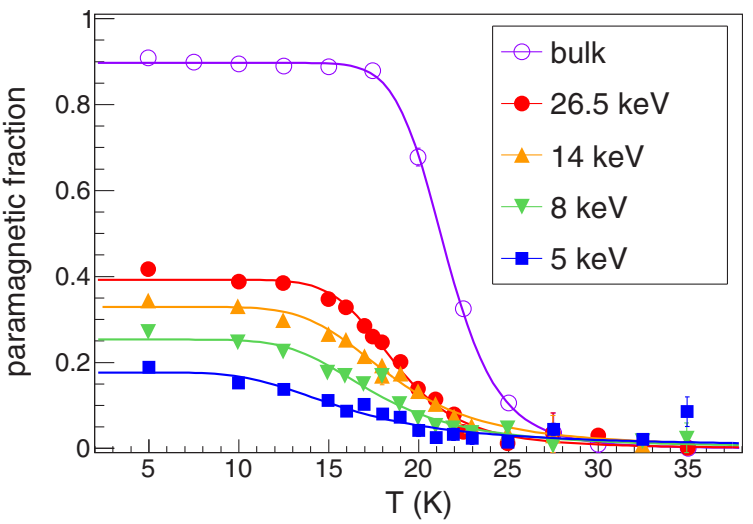

FIG. 3. (Color online) CdS (0001), neutral fraction $f_{\mathrm{Mu}}(T)$ as a function of temperature $T$ for different implantation energies. The bulk data are for the virgin sample, and the LE- $\mu$ SR data are for the etched sample. Solid lines are fits of Eq. (2) to the data to determine the shallow Mu ionization energy.

reduction of the $\mathrm{Mu}$ amplitudes even at highest implantation energies is likely to be caused by defects which are still present closer to the surface after the etching procedure.

The neutral fraction $f_{\mathrm{Mu}}(T)$ as a function of temperature is shown in Fig. 3, where the $\mu \mathrm{SR}$ asymmetry spectra were fit
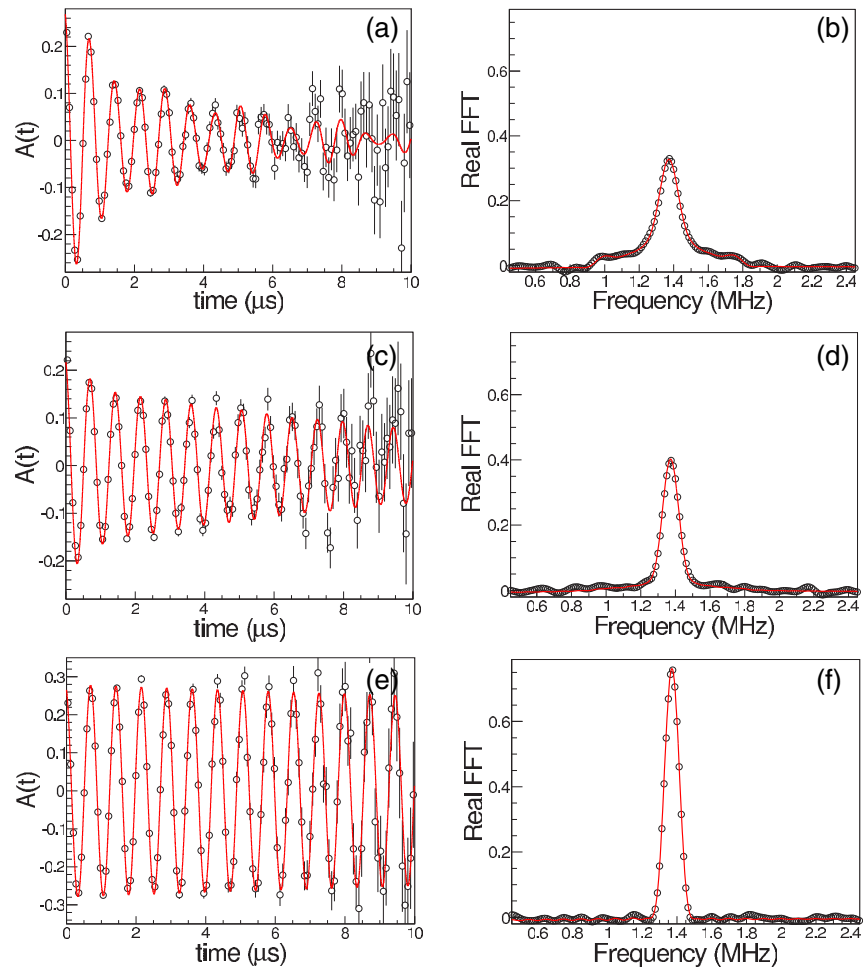

FIG. 4. (Color online) $\mathrm{ZnO}$ (0001), 10-mT applied parallel to a $\langle 0001\rangle$ direction, $\mu \mathrm{SR}$ asymmetry spectra $A(t)$, and corresponding real part of fast Fourier transform (Real FFT) of one of the positron counters. (a) and (b) $10 \mathrm{~K}$, implantation energy $17.5 \mathrm{keV}$ ( $\langle\mathrm{z}\rangle \sim 82 \mathrm{~nm})$. (c) and (d) $10 \mathrm{~K}$, implantation energy $2.5 \mathrm{keV}(\langle\mathrm{z}\rangle \sim$ $15 \mathrm{~nm}$ ). (e) and (f) $60 \mathrm{~K}$, implantation energy $17.5 \mathrm{keV}$. The solid red lines are fits to the data as described in the text, using the program MUSRFIT [53]. 
with Eq. (4) to determine the asymmetries $A_{\mathrm{D}}(T)$ and $A_{\mathrm{Mu}}(T)$. The decreasing neutral fraction with decreasing implantation energy below $26.5 \mathrm{keV}$ is a characteristic normally observed in insulators and semiconductors [55]. This is attributed to the fact that a substantial fraction of $\mathrm{Mu}$ is formed by those thermalized $\mu^{+}$which may capture one of the excess electrons generated in its own ionization track (so-called delayed $\mathrm{Mu}$ formation). The lower the energy the lower the number of track electrons, which reduces the $\mathrm{Mu}$ formation probability. Typically, this delayed $\mathrm{Mu}$ formation saturates if the stopping depth-i.e., the track length-of the $\mu^{+}$is of the order of hundred nanometer [55]. This length scale fits to earlier observations where the analysis of $\mu$ SR experiments with applied electric fields on bulk insulators suggested a similar length scale for delayed Mu formation [56]. Bulk $\mu$ SR experiments on $\mathrm{CdS}$ with an applied electric field showed that the recombination of a $\mu^{+}$with a track electron is highly suppressed at relatively weak electric fields of about $8 \mathrm{kV} / \mathrm{cm}$ [57]. As we will show below, the electric fields due to band bending in $\mathrm{CdS}$ at mean implantation depths $\langle\mathrm{z}\rangle<40 \mathrm{~nm}$ (implantation energy $<5 \mathrm{keV}$ ) are $6-8 \mathrm{kV} / \mathrm{cm}$. This means that the near-surface electric field additionally suppresses $\mathrm{Mu}$ formation.

The neutral fraction begins to drop at lower temperature the closer the muons stop to the surface. This reflects the decrease of the Mu ionization energy on approaching the surface and will be discussed in Sec. III C.

\section{B. $\mathrm{ZnO}$}

Compared to the $\mathrm{CdS}$ data, the shallow $\mathrm{Mu}$ lines in $\mathrm{ZnO}$ at $10 \mathrm{~K}$ are significantly broadened and unresolved, as shown in Figs. 4 and 5. The 10-K data in Figs. 4(a) and 4(c) were fit with five frequencies and fixed splitting of the shallow $\mathrm{Mu}$ lines, and the 60-K data in Fig. 4(e)-where shallow $\mathrm{Mu}$ is ionized-were fit with a single exponentially relaxing component. The bulk data are very similar to the $17.5 \mathrm{keV}$

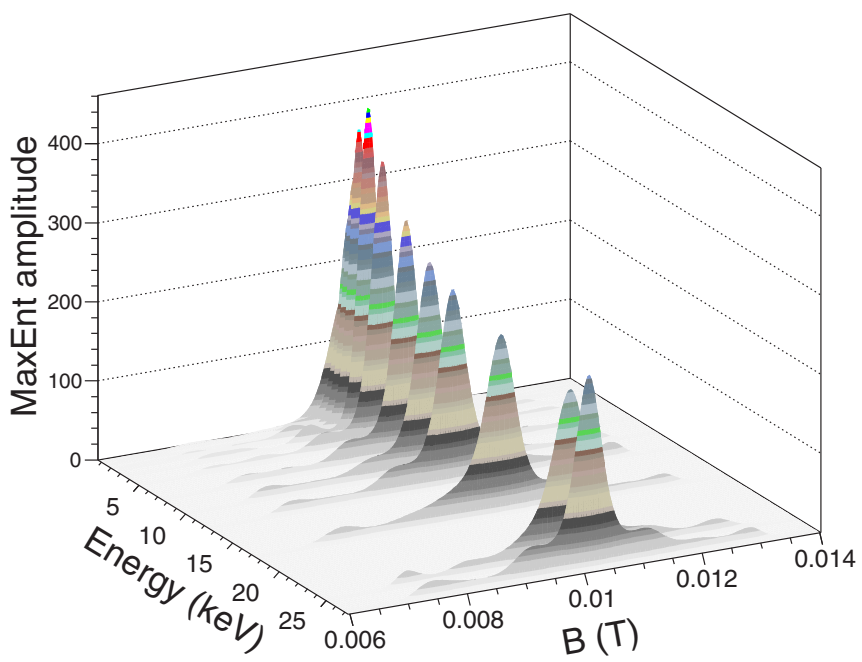

FIG. 5. (Color online) $\mathrm{ZnO}$ (0001), $10 \mathrm{~K}$, maximum entropy $[51,52]$ spectra as a function of implantation energy. On lowering, the implantation energy the diamagnetic peak increases at the expense of the shallow $\mathrm{Mu}$ satellite peaks. data of Fig. 4(a), also revealing unresolved shallow Mu lines. Figure 5 shows the frequency spectra at $10 \mathrm{~K}$ as a function of implantation energy, obtained by a maximum entropy fit of the time domain data [51,52]. Similar to the CdS data a clear increase of the diamagnetic line at the expense of the shallow $\mathrm{Mu}$ fraction is visible at decreasing implantation energies. We attribute this as well to the decreasing probability for delayed $\mathrm{Mu}$ formation due to the decreasing number of track electrons, and the presence of an electric field at the surface due to band bending. The higher maximum entropy amplitude of the diamagnetic line at $27 \mathrm{keV}$ compared to $25 \mathrm{keV}$ has its origin in the slightly more narrow line width of the diamagnetic signal at $27 \mathrm{keV}$ (the integral of the line, which equals the asymmetry $A_{\mathrm{D}}$ of the diamagnetic signal, is the same for both energies).

For the determination of the ionization energies at different depths, we use Eq. (5) to fit the $\mu$ SR asymmetry spectra, and we plot the relaxation rate $\lambda$ as a function of temperature and implantation energy. This is shown in Fig. 6 for $\mathrm{ZnO}$ and the $\mathrm{Au} / \mathrm{ZnO}$ Schottky barrier. The absolute value of $\lambda$ is proportional to the $\mathrm{Mu}$ fraction $f_{\mathrm{Mu}}(T)$ and Eq. (6) has been used to fit the data of Fig. 6. The relaxation rates in $\mathrm{Au} / \mathrm{ZnO}$ are generally smaller compared to $\mathrm{ZnO}$ because of muons stopping in the Au layer which contribute to the diamagnetic signal. Similar to CdS, the relaxation rate, i.e., the neutral fraction, begins to drop at lower temperatures the closer the muons stop at the surface/interface. This is again a manifestation of the decreasing ionization energy.
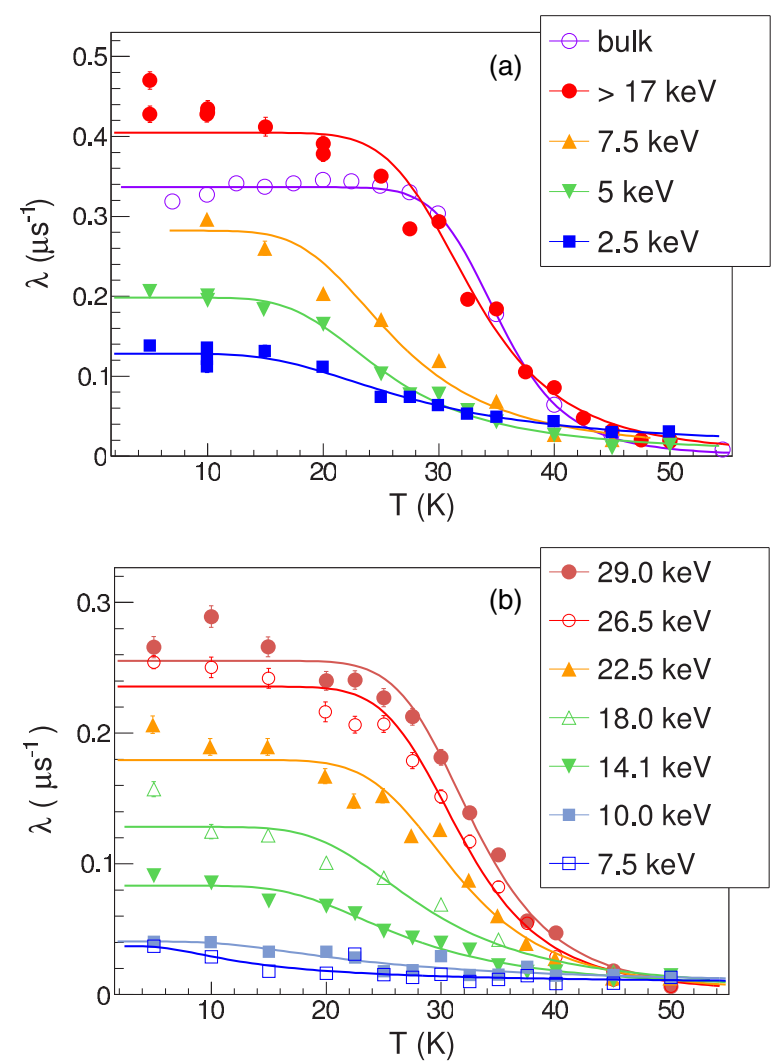

FIG. 6. (Color online) (a) $\mathrm{ZnO}$ (0001), single component exponential depolarization rate $\lambda$ as a function of temperature for different implantation energies. Solid lines are fits of Eq. (6) to the data to determine the shallow Mu ionization energy. (b) 20-nm Au on top of $\mathrm{ZnO}$ (0001). 

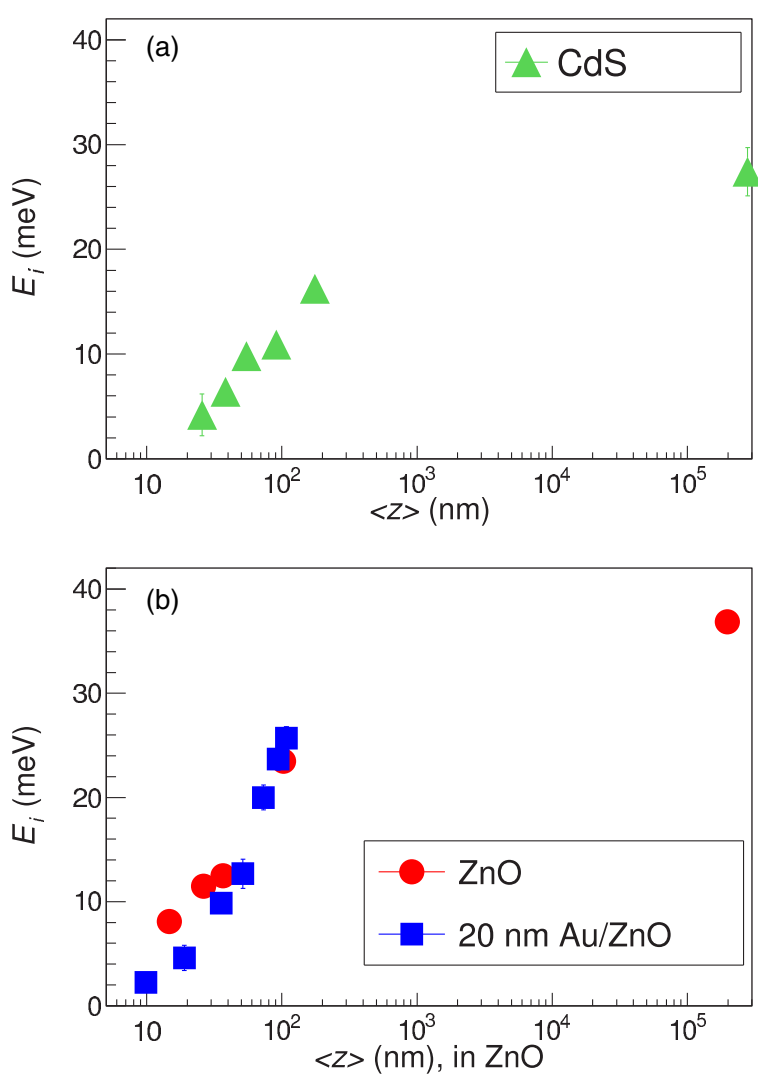

FIG. 7. (Color online) Ionization energy $E_{i}$ as a function of mean implantation depth $\langle z\rangle$ in (a) $\mathrm{CdS}$ and (b) $\mathrm{ZnO}$ and $\mathrm{Au} / \mathrm{ZnO}$ sample. Note that mean depths of $\langle z\rangle>200 \mathrm{~nm}$ and $\langle z\rangle \lesssim 100 \mu \mathrm{m}$ are experimentally not accessible due to the lack of muon beams with energies between $30 \mathrm{keV}$ and $\sim 1 \mathrm{MeV}$.

\section{Depth dependence of the ionization energy of shallow muonium in $\mathrm{CdS}$ and $\mathrm{ZnO}$}

The depth dependence of the ionization energies in $\mathrm{CdS}$ and in $\mathrm{ZnO}, \mathrm{Au} / \mathrm{ZnO}$, are shown in Fig. 7. Our bulk values $(\langle z\rangle \sim 300 \mu \mathrm{m})$ are in agreement with literature data. At the maximum accessible mean depth in LEM of $\sim 180 \mathrm{~nm}$, the ionization energy is already clearly reduced compared to the bulk value. This reduction is enhanced on approaching the surface, indicating an increase of the internal electric field. In Fig. 7(b), $\langle z\rangle$ denotes the mean depth with respect to the surface in $\mathrm{ZnO}$, and to the metal-semiconductor interface in $\mathrm{Au} / \mathrm{ZnO}$. At the $\mathrm{Au} / \mathrm{ZnO}$ interface the reduction of $E_{i}$ is larger compared to $\mathrm{ZnO}$ on a length scale of about $100 \mathrm{~nm}$. This can be attributed to a larger shift of the $\mathrm{ZnO}$ electronic bands at the interface due to the contact to the Au layer, causing a larger band bending, i.e., an enhanced electric field.

The $\mathrm{ZnO}$ data suggest a convergence with the bulk ionization energy at a depth of $\sim 0.5 \mu \mathrm{m}$, whereas in CdS, this length scale appears to be larger $(>1 \mu \mathrm{m})$. The room-temperature resistivity of the $\mathrm{ZnO}$ wafers is $10 \Omega \mathrm{cm}$, which is hundred times smaller than the resistivity of the CdS wafer. If we assume that this is caused by a hundred times higher free charge carrier concentration $n$ in $\mathrm{ZnO}$, the Debye length $L_{D}=$ $\sqrt{\varepsilon_{r} \varepsilon_{0} k_{B} T /\left(e^{2} n\right)}$ at room temperature, which is a measure of the depth of the band bending region, is expected to be

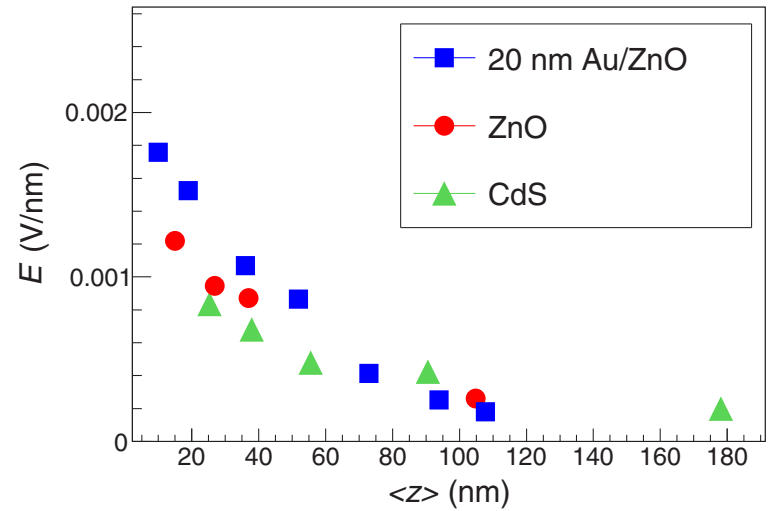

FIG. 8. (Color online) Calculated electric field $E$ as a function of mean depth $\langle z\rangle$ in $\mathrm{CdS}, \mathrm{ZnO}$, and $\mathrm{Au} / \mathrm{ZnO}$.

about ten times smaller in $\mathrm{ZnO}$. The estimated Debye length of $\sim 0.5 \mu \mathrm{m}$ in $\mathrm{ZnO}$ at $\sim 30 \mathrm{~K}$ implies a low temperature charge carrier concentration of $n \sim 5 \times 10^{12} \mathrm{~cm}^{-3}$, which is consistent with literature data [58]. The low temperature charge carrier concentration in CdS is then expected to be in the $10^{10} \mathrm{~cm}^{-3}$ range to obtain a Debye length of the order of $\mu \mathrm{m}$.

Using the data of Fig. 7 and the simple one-dimensional model described in Appendix, the electric field as a function of $\langle z\rangle$ can be calculated and is shown in Fig. 8. For $\mathrm{ZnO}$ and $\mathrm{Au} / \mathrm{ZnO}$ data $\langle z\rangle$ means again the distance to the surface $(\mathrm{ZnO})$, or to the $\mathrm{Au} / \mathrm{ZnO}$ interface. The increase of the electric field close to the $\mathrm{Au} / \mathrm{ZnO}$ interface due to enhanced band bending is clearly visible. As described in Sec. I a linear increase of the electric field toward the surface/interface is expected. However, the data indicate a deviation from linear dependence, with a faster increase of the field the closer the muons stop to the surface/interface. This could have its origin in the broad stopping distribution of the muons (see Fig. 1): in our simple analysis, we effectively determine an "averaged" ionization energy. The larger the implantation energy the larger the range for the averaging, which may cause the observed deviation from linear dependence.

\section{DISCUSSION}

As we noted in Sec. I the binding energy at mean depths $\langle z\rangle>10 \mathrm{~nm}$ is marginally affected by modifications of the wave function at the semiconductor surface, position dependent effective masses and dielectric constants, and dielectric confinement. The observed changes on $E_{i}$ in the depth range of our low-energy $\mu$ SR study $(10 \mathrm{~nm}<\langle z\rangle<200 \mathrm{~nm}$ ) can be naturally explained by assuming the presence of an electric field due to band bending. It is then the Poole-Frenkel effect which causes the reduction of $E_{i}$, and this allows us to relate the depth-dependent $E_{i}$ to the electric field profile.

Our results represent the first depth profiling of the ionization energy of a solitary hydrogenlike impurity state over a range of about $200 \mathrm{~nm}$ by means of a local probe technique. In this context, local probe means that the probe resides at an interstitial or substitutional side within the sample, where it "observes" its local environment on a nanometer scale, such as e.g., $\mu$ SR, NMR, $\beta$-NMR, ESR, PAC, or Moessbauer spectroscopy. The determination of the depth profile of $E_{i}$ 
at semiconductor surfaces or interfaces by low-energy $\mu \mathrm{SR}$ requires the detectability of the corresponding muonium states, e.g., semiconductors with doping levels $\lesssim 10^{17} \mathrm{~cm}^{-3}$, and not too high defect concentration. In Sec. III A, we showed that a significant fraction of $\mathrm{Mu}$ in semiconductors is due to delayed capture of an electron from the muon's ionization track, where electrons from the track up to distances of 50-100 nm can be captured. Assuming that the muon electron capture probability is reduced in the presence of defects-because defects in semiconductors usually act as recombination centers for excess carriers [59] — a rough estimate for the tolerable defect concentration is given by the requirement that there are no defects in a volume of $\sim(50 \mathrm{~nm})^{3}$ surrounding the stopped muon. This means that the defect concentration should not exceed $\sim 10^{16} \mathrm{~cm}^{-3}$.

The determination of the electric-field profile from the depth-dependent change in ionization energy is an indirect method, but it has the advantage that the sample can be studied as it is. This is different to a "surface technique" such as Kelvin probe microscopy (KPM), which can directly measure the potential profile at the surface of cleaved samples. It has been frequently used in semiconductor studies on $p n$ junctions, heterostructures, transistors, and solar cells [60], with a spatial resolution in the nanometer range. Unlike $\mu \mathrm{SR}$, it cannot provide information about the ionization energy of single impurities.

The presented procedure offers the interesting possibility to study the characteristics of shallow impurities in the presence of other dopants: the implantation of solitary impurities allows to indirectly sense the intrinsic charge carrier concentration due its effect on the band bending close to the surface or an interface. The direction of band bending is not accessible here because the changes on the ionization energy only depend on the absolute value of the electric field. Since muon spin rotation is contactless and does not need the application of an electric potential at the surface it provides direct information about intrinsic properties of the semiconductor.

The simplification of the one-dimensional model is well justified if we assume that the minimum of the ionization potential in one direction is the dominant effect on the measured change of the ionization energy. The full threedimensional (3D) case is discussed by Martin and co-workers for deep impurity levels [18], where the authors calculate the electron emission rate from the impurity state in the $3 \mathrm{D}$ case. For example, the electron emission rate is diminshed in positive $z$ direction in the situation sketched in Fig. 9. On the other hand, the electron emission rate is increased by phononassisted tunneling and pure quantum mechanical tunneling. For deep levels, pure tunneling becomes important only at very high fields $\left(\sim 10^{7} \mathrm{~V} / \mathrm{cm}\right)$, and a significant emission rate enhancement occurs only for fields $\gg 10^{4} \mathrm{~V} / \mathrm{cm}$ [18], which are much larger than the electric fields in our experiment.

\section{CONCLUSIONS}

In summary, we have shown by means of low-energy $\mu$ SR that the ionization energy of single shallow hydrogenlike muonium states in $\mathrm{CdS}, \mathrm{ZnO}$, and $\mathrm{Au} / \mathrm{ZnO}$ decreases on approaching the semiconductor surface or interface. Compared to the value measured deep in the bulk at $\langle z\rangle \sim 300 \mu \mathrm{m}$ the

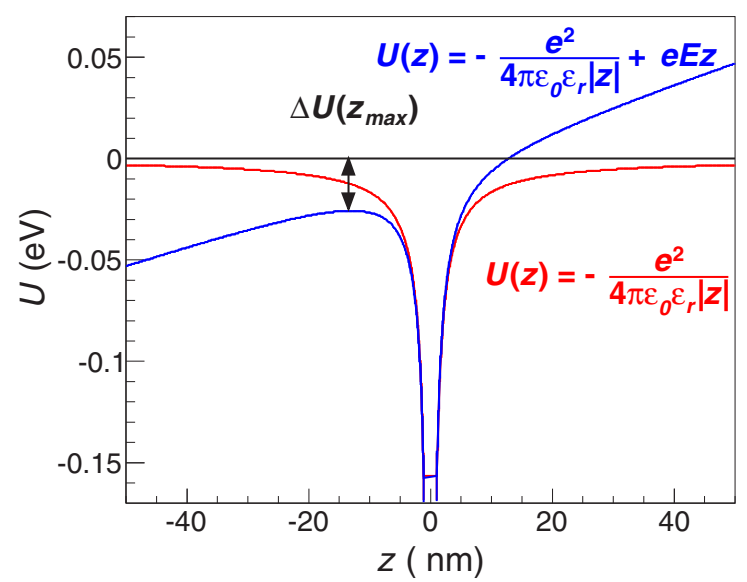

FIG. 9. (Color online) Electric potential energy $U(z)$ as a function of $z$ for a pointlike charge with and without electric field $E$. For the calculation, we used the relative permittivitiy of $\varepsilon_{r}=8.5$ for $\mathrm{ZnO}$ and $E=10 \mathrm{kV} / \mathrm{cm}=0.001 \mathrm{~V} / \mathrm{nm}$. The double-arrow indicates the position of the local maximum $z_{\max }$ of $U(z)$ in the presence of an electric field and the reduction $\Delta U\left(z_{\max }\right)$ of the ionization energy.

ionization energy is diminshed by $\sim 10 \mathrm{meV}$ at mean depths of $100-150 \mathrm{~nm}$, and further reduced by up to $25-30 \mathrm{meV}$ at a depth of $10 \mathrm{~nm}$. This reduction is attributed to the presence of electric fields (Poole-Frenkel effect) near the surface/interface due to band bending. Other mechanisms potentially able to cause a change of the ionization energy (modifications of the wave function, position dependent effective masses and dielectric constants, dielectric confinement) can be excluded in the investigated depth range. Using a simple one-dimensional model allowed to determine the near-surface/interface electric field profile inside the sample. This kind of investigation recently revealed the presence of a shallow hydrogen donor state in $\mathrm{SrTiO}_{3}$ with decreasing ionization energy at the surface [61]. It can be extended to semiconductors or semiconductor heterostructures with not too high doping levels $\left(\lesssim 10^{17} \mathrm{~cm}^{-3}\right)$ and defect concentrations $\left(\lesssim 10^{16} \mathrm{~cm}^{-3}\right)$.

\section{ACKNOWLEDGMENTS}

We gratefully acknowledge the technical support of H. P. Weber, and the contributions of D. G. Eshchenko in the setup of the LEM facility. We thank J. M. Campos Gil, H. V. Alberto, and R. C. Vilão for stimulating discussions. The $\mu$ SR measurements were performed at the Swiss Muon Source $\mathrm{S} \mu \mathrm{S}$, Paul Scherrer Institut, Villigen, Switzerland.

\section{APPENDIX: CALCULATION OF THE ELECTRIC FIELD}

For the calculation of the electric field at the surface, we use a one-dimensional approximation since we are only interested in the maximum reduction of the ionization energy in the presence of an electric field. The electric potential energy $U$ of a point charge $e$ in the presence of an electric field $E$ along the direction $z$ can be written as

$$
U(z)=-\frac{e^{2}}{4 \pi \varepsilon_{0} \varepsilon_{r}|z|}+e E z,
$$




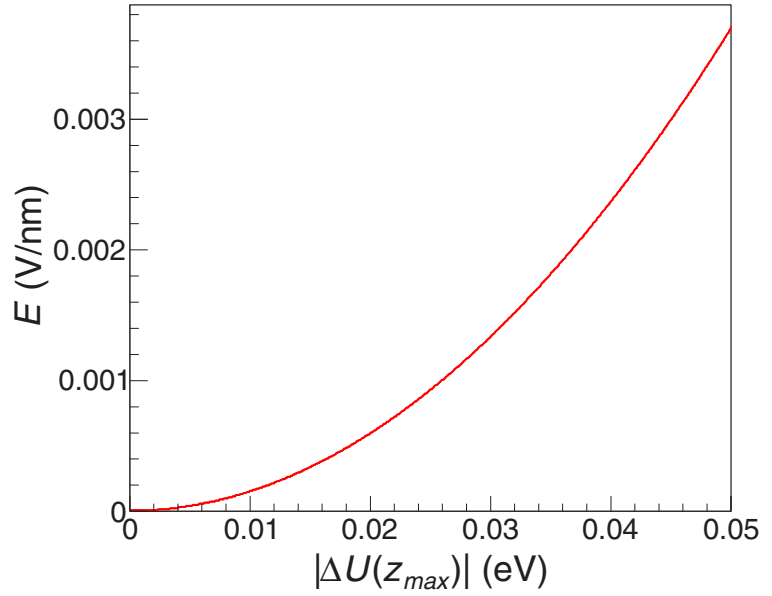

FIG. 10. (Color online) Electric field $E$ as a function of $\Delta U\left(z_{\max }\right)$, according to Eq. (A3), using the relative permittivity of $\mathrm{ZnO}, \varepsilon_{r}=8.5$. where $\varepsilon_{0}$ is the vacuum permittivity, and $\varepsilon_{r}$ is the relative permittivity of the semiconductor. The change in ionization energy $E_{i}$ is given by $\Delta U\left(z_{\max }\right)$, as indicated in Fig. 9. It can be easily derived from Eq. (A1) [16]:

$$
\Delta U\left(z_{\max }\right)=-e \sqrt{\frac{e E}{\pi \varepsilon_{0} \varepsilon_{r}}} .
$$

Solving Eq. (A2) for $E$, we can write the electric field as a function of the reduction of ionization energy $\Delta U\left(z_{\max }\right)$ (see Fig. 10):

$$
E\left(\Delta U\left(z_{\max }\right)\right)=\frac{\pi \varepsilon_{0} \varepsilon_{r}\left[\Delta U\left(z_{\max }\right)\right]^{2}}{e^{3}} .
$$

We calculated the electric fields shown in Fig. 8 with Eq. (A3), where we used $\Delta U\left(z_{\max }\right)=E_{i}(z>200 \mu \mathrm{m})-E_{i}(z)$, and $\varepsilon_{r}=8.9$ for $\mathrm{CdS}$ and $\varepsilon_{r}=8.5$ for $\mathrm{ZnO}$.
[1] P. M. Koenraad and M. E. Flatté, Nat. Mater. 10, 91 (2011).

[2] H. Sellier, G. P. Lansbergen, J. Caro, S. Rogge, N. Collaert, I. Ferain, M. Jurczak, and S. Biesemans, Phys. Rev. Lett. 97, 206805 (2006).

[3] S. Perraud, K. Kanisawa, Z.-Z. Wang, and T. Fujisawa, Phys. Rev. Lett. 100, 056806 (2008).

[4] K. Teichmann, M. Wenderoth, S. Loth, R. G. Ulbrich, J. K. Garleff, A. P. Wijnheijmer, and P. M. Koenraad, Phys. Rev. Lett. 101, 076103 (2008).

[5] A. P. Wijnheijmer, J. K. Garleff, K. Teichmann, M. Wenderoth, S. Loth, R. G. Ulbrich, P. A. Maksym, M. Roy, and P. M. Koenraad, Phys. Rev. Lett. 102, 166101 (2009).

[6] G. Bastard, Wave Mechanics Applied to Semiconductor Heterostructures (Editions de Physique, Les Ulis, 1988).

[7] C. Mailhiot, Y.-C. Chang, and T. C. McGill, Phys. Rev. B 26, 4449 (1982).

[8] J. D. Levine, Phys. Rev. 140, A586 (1965).

[9] T.-F. Jiang and Y. Shan, J. Phys. C 18, 3399 (1985).

[10] M. Diarra, Y.-M. Niquet, C. Delerue, and G. Allan, Phys. Rev. B 75, 045301 (2007).

[11] A. J. Peter, Int. J. Mod. Phys. B 23, 5109 (2009).

[12] J. Zak and W. Zawadzki, Phys. Rev. 145, 536 (1966).

[13] J. M. Luttinger and W. Kohn, Phys. Rev. 97, 869 (1955).

[14] W. Kohn, in Solid State Physics, edited by Frederick Seitz and David Turnbull (Academic Press, New York, 1957), Vol. 5, pp. 257-320.

[15] S. M. Sze and K. K. Ng, Physics of Semiconductor Devices, 3rd ed. (John Wiley \& Sons, Inc., Hoboken, NJ, USA, 2006).

[16] J. Frenkel, Phys. Rev. 54, 647 (1938).

[17] J. L. Hartke, J. Appl. Phys. 39, 4871 (1967).

[18] P. A. Martin, B. G. Streetman, and K. Hess, J. Appl. Phys. 52, 7409 (1981).

[19] B. D. Patterson, Rev. Mod. Phys. 60, 69 (1988).

[20] K. H. Chow, B. Hitti, and R. F. Kiefl, in Semiconductors and Semimetals, Vol. 51A, edited by M. Stavola (Academic Press, San Diego and London, 1998).

[21] S. F. J. Cox, J. Phys.: Condens. Matter 15, 1727 (2003).
[22] S. F. J. Cox, Rep. Prog. Phys. 72, 116501 (2009).

[23] A. Yaouanc and P. Dalmas de Réotier, Muon Spin Rotation, Relaxation, and Resonance: Applications to Condensed Matter (Oxford University Press, Oxford, UK, 2011).

[24] C. Kilic and A. Zunger, Appl. Phys. Lett. 81, 73 (2002).

[25] C. G. Van de Walle and J. Neugebauer, Nature (London) 423, 626 (2003).

[26] C. G. Van de Walle, Phys. Rev. Lett. 85, 1012 (2000).

[27] S. F. J. Cox, E. A. Davis, S. P. Cottrell, P. J. C. King, J. S. Lord, J. M. Gil, H. V. Alberto, R. C. Vilão, J. Piroto Duarte, N. Ayres de Campos, A. Weidinger, R. L. Lichti, and S. J. C. Irvine, Phys. Rev. Lett. 86, 2601 (2001).

[28] E. A. Davis, S. F. J. Cox, R. L. Lichti, and C. G. Van de Walle, Appl. Phys. Lett. 82, 592 (2003).

[29] D. M. Hofmann, A. Hofstaetter, F. Leiter, H. Zhou, F. Henecker, B. K. Meyer, S. B. Orlinskii, J. Schmidt, and P. G. Baranov, Phys. Rev. Lett. 88, 045504 (2002).

[30] M. D. McCluskey, S. J. Jokela, K. K. Zhuravlev, P. J. Simpson, and K. G. Lynn, Appl. Phys. Lett. 81, 3807 (2002).

[31] E. V. Lavrov, J. Weber, F. Börrnert, C. G. Van de Walle, and R. Helbig, Phys. Rev. B 66, 165205 (2002).

[32] J. M. Gil, H. V. Alberto, R. C. Vilão, J. Piroto Duarte, P. J. Mendes, L. P. Ferreira, N. Ayres de Campos, A. Weidinger, J. Krauser, Ch. Niedermayer, and S. F. J. Cox, Phys. Rev. Lett. 83, 5294 (1999).

[33] J. M. Gil, H. V. Alberto, R. C. Vilão, J. Piroto Duarte, N. Ayres de Campos, A. Weidinger, J. Krauser, E. A. Davis, S. P. Cottrell, and S. F. J. Cox, Phys. Rev. B 64, 075205 (2001).

[34] K. Shimomura, R. Kadono, K. Ohishi, M. Mizuta, M. Saito, K. H. Chow, B. Hitti, and R. L. Lichti, Phys. Rev. Lett. 92, 135505 (2004).

[35] C. G. Van de Walle, Physica B: Condens. Matter 376-377, 1 (2006).

[36] R. L. Lichti, K. H. Chow, and S. F. J. Cox, Phys. Rev. Lett. 101, 136403 (2008).

[37] W. Eckstein, Computer Simulation of Ion-Solid Interactions (Springer, Berlin, Heidelberg, New York, 1991). 
[38] E. Morenzoni, H. Glückler, T. Prokscha, R. Khasanov, H. Luetkens, M. Birke, E. M. Forgan, Ch. Niedermayer, and M. Pleines, Nucl. Instrum. Methods Phys. Res., Sect. B 192, 254 (2002).

[39] E. Morenzoni, H. Glückler, T. Prokscha, H. P. Weber, E. M. Forgan, T. J. Jackson, H. Luetkens, Ch. Niedermayer, M. Pleines, M. Birke, A. Hofer, J. Litterst, T. Riseman, and G. Schatz, Physica B: Condens. Matter 289-290, 653 (2000).

[40] T. Prokscha, E. Morenzoni, K. Deiters, F. Foroughi, D. George, R. Kobler, A. Suter, and V. Vrankovic, Nucl. Instrum. Methods Phys. Res., Sect. A 595, 317 (2008).

[41] D. R. Harshman, A. P. Mills, J. L. Beveridge, K. R. Kendall, G. D. Morris, M. Senba, J. B. Warren, A. S. Rupaal, and J. H. Turner, Phys. Rev. B 36, 8850 (1987).

[42] E. Morenzoni, F. Kottmann, D. Maden, B. Matthias, M. Meyberg, Th. Prokscha, Th. Wutzke, and U. Zimmermann, Phys. Rev. Lett. 72, 2793 (1994).

[43] T. Prokscha, E. Morenzoni, C. David, A. Hofer, H. Glückler, and L. Scandella, Appl. Surface Sci. 172, 235 (2001).

[44] E. Morenzoni, T. Prokscha, A. Suter, H. Luetkens, and R. Khasanov, J. Phys.: Condens. Matter 16, S4583 (2004).

[45] K. Seeger, Semiconductor Physics: An Introduction, 9th ed. (Springer, Berlin, Heidelberg, New York, 2004).

[46] S. F. J. Cox, J. L. Gavartin, J. S. Lord, S. P. Cottrell, J. M. Gil, H. V. Alberto, J. Piroto Duarte, R. C. Vilão, N. Ayres de Campos, D. J. Keeble, E. A. Davis, M. Charlton, and D. P. van der Werf, J. Phys.: Condens. Matter 18, 1079 (2006).

[47] M. Senba, J. Phys. B 38, 1305 (2005).

[48] J. M. Gil, J. Piroto Duarte, R. C. Vilão, H. V. Alberto, N. Ayres de Campos, and S. F. J. Cox, Physica B: Condens. Matter 404, 834 (2009).
[49] R. C. Vilão, A. G. Marinopoulos, R. B. L. Vieira, A. Weidinger, H. V. Alberto, J. Piroto Duarte, J. M. Gil, J. S. Lord, and S. F. J. Cox, Phys. Rev. B 84, 045201 (2011).

[50] R. Abela, A. Amato, C. Baines, X. Donath, R. Erne, D. C. George, D. Herlach, G. Irminger, I. D. Reid, D. Renker, G. Solt, D. Suhi, M. Werner, and U. Zimmermann, Hyperfine Interact. 120-121, 575 (1999).

[51] T. M. Riseman and E. M. Forgan, Physica B: Condens. Matter 326, 226 (2003).

[52] T. M. Riseman and E. M. Forgan, Physica B: Condens. Matter 326, 230 (2003).

[53] A. Suter and B. Wojek, Physics Procedia 30, 69 (2012).

[54] H. V. Alberto, R. C. Vilão, J. Piroto Duarte, J. M. Gil, A. Weidinger, J. S. Lord, and S. J. F. Cox, Phys. Rev. B 86, 035203 (2012).

[55] T. Prokscha, E. Morenzoni, D. G. Eshchenko, N. Garifianov, H. Glückler, R. Khasanov, H. Luetkens, and A. Suter, Phys. Rev. Lett. 98, 227401 (2007).

[56] D. G. Eshchenko, V. G. Storchak, J. H. Brewer, G. D. Morris, S. P. Cottrell, and S. F. J. Cox, Phys. Rev. B 66, 035105 (2002).

[57] D. G. Eshchenko, V. G. Storchak, S. P. Cottrell, and S. F. J. Cox, Phys. Rev. B 68, 073201 (2003).

[58] A. Y. Polyakov, N. B. Smirnov, A. V. Govorkov, E. A. Kozhukhova, S. J. Pearton, D. P. Norton, A. Osinsky, and Amir Dabiran, J. Electron. Mater. 35, 663 (2006).

[59] D. K. Schroder, Semiconductor Material and Device Characterization, 3rd ed. (Wiley, Hoboken, NJ, USA, 2006).

[60] W. Melitz, J. Shen, A. C. Kummel, and S. Lee, Surface Sci. Rep. 66, 1 (2011).

[61] Z. Salman, T. Prokscha, A. Amato, E. Morenzoni, R. Scheuermann, K. Sedlak, and A. Suter, Phys. Rev. Lett. 113, 156801 (2014). 